\title{
Evaluation of Vertical Bone Augmentation Using a Titanium Ring Retained in Simultaneous Implants With Early Exposure: A Pilot Study in Dogs
}

Qi-Qi Liu

Southwest Medical University

Jian Yang

Southwest Medical University

Lin-Jia Lai

Southwest Medical University

Pei-Zhao Peng

Southwest Medical University

Mao-Yun Zeng

Southwest Medical University

KeYu (D 65968999@qq.com)

Southwest Medical University

\section{Research Article}

Keywords: animal experiment, early exposure, vertical bone augmentation, titanium ring, implant

Posted Date: October 22nd, 2021

DOI: https://doi.org/10.21203/rs.3.rs-962303/v1

License: (c) (i) This work is licensed under a Creative Commons Attribution 4.0 International License.

Read Full License 


\section{Abstract}

To evaluate the performance of titanium rings retained in implants placed simultaneously for vertical bone augmentation with early exposure. Twenty-four bone level tapered dental implants were inserted in the premolar areas of the mandible with standardized, $3.5 \mathrm{~mm}$ deep, vertical bone defects in four beagle dogs, and titanium rings containing a mixture of autogenous bone chips and Bio-oss were retained in the implants for vertical bone augmentation. Three weeks after the operation, all titanium rings were exposed, one titanium ring from each mandible of each dog was removed, and the other titanium ring was left in place with daily flushing. After 2 months, the dogs were sacrificed, the height of vertical bone augmentation was measured, and bone samples containing the implants were harvested and analyzed. The median (minimum-maximum) bone augmentation height (BAH) was 2.46 (2.18-2.62) $\mathrm{mm}$ on the buccal side and 2.80 (2.66-3.02) $\mathrm{mm}$ on the lingual side in the removal group; however, the BAH was 0.64 $(0.32-0.92) \mathrm{mm}$ and $0.70(0.22-1.22) \mathrm{mm}$ in the holding group. The bone volume/total volume (BV/TV) of the increased bone was $80.07 \%$ (72.64\%-87.89\%) with micro-CT analysis in the removal group, ideal bone osseointegration formed, and new bone was observed in the hard tissue sections. Within its limitations, the use of titanium rings retained in implants was found to be a reliable alternative method for vertical bone augmentation, and removing titanium rings is better for bone augmentation when early exposure occurs.

\section{Introduction}

Alveolar bone defects resulting from either periodontal disease or dental trauma will increase the difficulty of implant placement operations ${ }^{1}$. To overcome unfavorable changes in the height and width of alveolar bone, a plethora of bone augmentation techniques have been adopted for implant placement either in a simultaneous or a consecutive approach ${ }^{2,3}$. Among them, vertical bone augmentation is a more difficult way to acquire adequate alveolar ridges for successful dental implant placement ${ }^{4,5}$. In most cases, a healing period of at least 6 months has been suggested to ensure successful augmentation and accurate implant positioning during the second surgery ${ }^{6}$.

A variety of techniques have been used for vertical bone augmentation in patients with atrophic alveolar ridges, such as distraction osteogenesis, autogenous block grafting (bone block or bone ring), and guided bone regeneration (GBR) using titanium mesh or expanded-polytetrafluoroethylene (e-PTFE) membranes ${ }^{7-9}$. Resorption of the bone segment and inclination of the distractor will decrease the efficacy of distraction osteogenesis ${ }^{10}$, and the mobilized fragment should be more than $4 \mathrm{~mm}$ thick ${ }^{11}$. High technical sensitivity and potential risks, including nerve damage or hypoesthesia bone segment displacement, premature consolidation, distractor device instability, transport bone segment or mandible base fracture, and segment nonunion, will limit the popularity of distraction osteogenesis ${ }^{12}$. On the other hand, although autogenous block grafts are considered the gold standard for vertical bone augmentation, the drawbacks of autogenous block grafts are also obvious, including restricted donor sites, possible harvesting morbidity, reports of unpredictable resorption and poor patient acceptance ${ }^{13}$. 
GBR with titanium mesh is an effective alternative method for vertical bone augmentation ${ }^{14}$. The successful reconstruction of large discontinuity osseous defects by a titanium mesh was reported in $1985^{15}$. This metal has been used extensively in numerous surgical applications because it is highly reactive and has a low weight and can be easily passivated to form a protective oxide layer, which accounts for its high corrosion resistance ${ }^{16}$. A low density of titanium provides high strength and lightweight dental implant bone augmentation barrier membrane material ${ }^{17}$. Titanium mesh has a definite strength that can guide the control of the contour profile of regenerated bone and maintain a relatively stable space compared to conventional barrier membranes ${ }^{18}$. Moreover, the good biocompatibility of titanium mesh can avoid any immune response ${ }^{14}$. Previous studies have shown that when titanium mesh is applied as a barrier membrane, bone augmentation can reach up to $10 \mathrm{~mm}$ vertically and horizontally, and the amount of long-term bone resorption is small ${ }^{19,20}$.

During the early phase of healing, sufficient fixation of the graft and barrier membrane is essential to achieve early revascularization and graft integration. In addition, micromovement has been demonstrated to result in the development of fibrous tissue rather than bone tissue ${ }^{21,22}$. As a type of GBR membrane, titanium mesh adapts to various bone defects through bending and shaping, which may increase the time of surgery and generate sharp edges ${ }^{14}$. Moreover, the space maintenance capacity of titanium mesh is based on self-superior positional fixation, which could be achieved by using a retentive pin, but the use of a retentive pin would make the implant surgery difficult and more cumbersome, regardless of whether it was implanted or removed ${ }^{18}$.

Based on several previous studies, the main complication of titanium mesh is postoperative exposure following wound dehiscence, at a rate of $14.8 \%-66 \%^{20,23-27}$. As another kind of nonresorbable barrier membrane of GBR, e-PTFE leads to a higher rate of infection once exposed ${ }^{28}$, but infection does not necessarily occur after titanium mesh exposure ${ }^{19,29}$. Furthermore, how to address postoperative exposure of titanium mesh is controversial ${ }^{17,30}$.

In view of these problems, we have developed an alternative titanium ring technique using a personalized titanium mesh printed by three-dimensional (3D) printing technology. The titanium ring was fixed by the implant through a retaining screw and could be personalized by 3D printing according to the shape of the bone defect, including horizontal or vertical bone defects. The aim of the present study was to assess the bone augmentation effect of the titanium ring technique and to compare different treatment methods (removing the titanium and removing the titanium with flushing) after early exposure after treating standardized bone defects in beagle dog mandibles.

\section{Methods}

Six male beagle dogs with a weight between 10 and $13 \mathrm{~kg}$ and a mean age of 14 months were used in the present study. The animals were housed in the Animal Facility for Oral and Maxillofacial Reconstruction and Regeneration Experiments, School of Stomatology, Southwest Medical University at 
Luzhou, China. The appearance, behavior, reactivity and social interactions of the dogs were monitored during the entire treatment period. Each animal was kept in a separate cage with a light:dark cycle of $12: 12 \mathrm{~h}$ and without excessive or potentially startling noises. This study was approved by the Animal Experiment Ethics Committee of Southwest Medical University, Luzhou, China (No. 2021-0528-1), and it is reported according to The ARRIVE (Animal Research: Reporting of In Vivo Experiments) guidelines. All methods were performed in accordance with the relevant guidelines and regulations.

\section{Titanium Ring and Retaining Screw}

The titanium ring of the present study was first designed by 3-matic Research (Release 11 for Windows, Materialise, Leuven, Belgium) following the creation of the original STL format file. A unique design feature of the titanium ring was that its inner ring was designed as a frustum of a cone with a central hole, which matched with the nonhex implant with a morse taper (Dentium Superline, Ltd, Gyeonggi-do, Korea) and retaining screw (Fig. 6). A pore size of $500 \mu \mathrm{m}$ was created at the top of the titanium ring, with no pores on the sides.

The 3D printer (M290, EOS, Munich, Germany) used a high-energy laser to melt the titanium metal powder into slices of the required three-dimensional shape, and then the sintering machine accumulated these slices layer by layer to obtain the required titanium ring. The retaining screw stemmed from the abutment screw (Dentium Superline, Ltd., Gyeonggi-do, Korea) and could easily secure the titanium ring to the implant after machining (Fig. 7). Subsequently, the final titanium ring and retaining screw were checked and polished. Before grafting in beagle dogs, all products were sterilized by high temperature and high pressure, reaching the requirements of a surveillance standard for sterilization (ISO 15883:2006).

\section{Surgical Procedures}

The study was designed as a pilot experimental study. All surgeries were performed under general anesthesia, and surgical interventions were performed under systemic anesthesia using pentobarbital sodium (i.m. $30 \mathrm{mg} / \mathrm{kg}$ ). In the first surgery, three premolars (P2-P4 per hemimandible) were atraumatically extracted by root separation from both sides of the mandible. All animals received $125 \mathrm{mg}$ Amoclav (clavulanic acid and amoxicillin, Hanvet Pet Health, Shanghai, China) as antibiotics intraoperatively and twice a day for 7 days after the surgery. Appropriate therapy was administered when the behavior of the animals indicated adverse reactions or pain. The sutures were removed 7 days after the surgery.

After 3 months of healing, the second surgery involved a midcrestal incision and full-thickness mucoperiosteal flap elevation. Two standard three-dimensional ridge defects of $7.2 \mathrm{~mm}$ (mesio-distal and buccal-lingual) to $3.5 \mathrm{~mm}$ (apico-coronal) were created on P2 and P4 of each side of the mandible. Following the drill protocol of the manufacturer, the first guide drill was navigated and fixed $5 \mathrm{~mm}$ below the bone defect, and then the final drill was used to enlarge the diameter of the hole to $2.8 \mathrm{~mm}$ at $50 \mathrm{rpm}$ while the autogenous bone particles were harvested. Bone level implants ( $\varnothing 3.6 \mathrm{~mm}$, length $8 \mathrm{~mm}$ ) were inserted in the hole to $5 \mathrm{~mm}$ to achieve adequate primary stability, with $3 \mathrm{~mm}$ exposed. A titanium ring 
was secured to the implant with a retaining screw, and a combination of deproteinized natural bovine bone mineral (DBBM) (Bio-oss, geistlish Pharma, Wolhusen, Switzerland) and autogenous bone particles was used to fill the void between the implant and the titanium ring. The flap was relaxed by releasing the periosteum and then sutured to allow for submerged healing of all implants.

Normal saline was used to irrigate the wound once a day for 3 weeks after surgery without applying anesthesia. Unfortunately, all titanium rings were exposed 3 weeks after the operation due to the dehiscence of the flap (Fig. 8). Subsequently, the experimental sites were randomly divided into two groups, and each group included p2 of one mandible and p4 on the other in the same beagle dog. In the titanium ring removal group, the small flap of soft tissue was elevated to remove the titanium ring, and then the soft tissue was sutured again after normal saline irrigation at 4 weeks. No surgical treatments were carried out in the titanium ring nonremoval group (holding group). However, daily flushing was still performed with normal saline.

Sample Preparation

After an osseointegration period of 3 months, the animals were euthanized by an intramuscular overdose of pentobarbital sodium $(100 \mathrm{mg} / \mathrm{kg})$. Block specimens of the entire implant and surrounding bone (mesiodistal $3 \mathrm{~mm}$ ) were harvested except for loose implants after elevating the full-thickness mucoperiosteal flap. Furthermore, the trimmed mandible bone blocks containing the implants were fixed in $4 \%$ paraformaldehyde and analyzed by means of micro-CT and histology.

\section{Micro-CT Analysis}

The biopsies were nondestructively examined first with a radiographic computed microtomography (micro-CT) system. The samples were placed in a vertical position to the direction of the radiation and scanned using a micro-CT system (Venus, VNC-102; PINGSENG Health care, Kunshan, China) as follows: $\mathrm{X}$-ray source, $80 \mathrm{kV}, 80 \mu \mathrm{A}$; distance from the source to the object, $90 \mathrm{~mm}$; distance from the source to the detector, $410 \mathrm{~mm}$ : slice thickness, $0.08 \mathrm{~mm}$; voxel size, $0.049 \times 0.049 \times 0.08 \mathrm{~mm}$. The micro-CT images $(270$ slices) were then reconstructed using 3D structural analysis software (Avatar; PINGSENG Health care, Kunshan, China). Threshold values were set for the segmentation between 5400 and 22800 for the implant and 2500 and 5400 for the bone tissue. The region of interest (ROI) was selected corresponding to the dimensions of the titanium ring, with a diameter of $7.2 \mathrm{~mm}$ and the base at $3 \mathrm{~mm}$ from the implant shoulder (V1). Bone directly under V1 with a diameter of $7.2 \mathrm{~mm}$ and a height of $5 \mathrm{~mm}$ was measured separately (V2). Then, the bone volume (BV, $\left.\mathrm{mm}^{3}\right), \mathrm{BV} /$ total volume (BV/TV), bone surface/TV (BS/TV) and bone mineral density $\left(\mathrm{BMD}, \mathrm{g} / \mathrm{mm}^{3}\right)$ were determined in $\mathrm{V} 1$. Only BMD was calculated in $\mathrm{V} 2$.

Histological Preparation

First, the bone samples were fixed for one week, and the fixation liquid was replaced overnight. Following rinsing in running tap water for 24 hours, trimming, and dehydrating in ascending concentrations of ethanol from $70-100 \%$, the bone samples were made transparent by using ETC-3 (Combine, Changsha, 
China) for less than 4 hours, and then the bone samples were processed for the production of undecalcified ground sections and embedded in a mixture of methylmethacrylate, dibutyl violet phthalate and benzoylperoxide. The embedded tissue blocks were cut buccolingually along the implant axis using a slow-speed rotating diamond serrated blade (LEICA1600 VC-50, Frankfurt, Germany) into approximately $0.08 \mathrm{~mm}$ thick ground sections. The sections were stained with toluidine blue and photographed under a light microscope equipped with a digital imaging system (CellSens; OLYMPUS, Tokyo, Japan).

\section{Statistical Analysis}

To quantify the effects of the titanium rings on the outcomes, absolute values were used. Total bone was defined as newly formed bone (including osteoid and woven bone) and residual graft bone. All of the quantitative variables were characterized using descriptive statistics (median, minimum and maximum) instead of means and standard deviations, and a normal distribution was not assumed considering the low sample size. Kruskal-Wallis nonparametric analysis of variance was applied to estimate the differences in BAH over the buccalingual aspects of the implants in the two groups. In addition, the difference in BAH over the two groups was evaluated by the Wilcoxon rank-sum test. The Wilcoxon signed-rank test was used to estimate the differences in BMD over the V1 and V2 and in BAH over the buccolingual aspects of the implant between the removal group and the holding group, respectively. $\mathrm{P}$ values $<0.05$ were considered statistically significant. The statistical analysis was conducted using SPSS for Windows Release 26.0, standard version (IBM SPSS, Chicago, IL, USA).

\section{Results}

\section{Clinical Observations}

The titanium ring fit perfectly into the prepared defective sockets without any need for recontouring or adjustment, and the implants were installed passively to gain initial stability. All six animals survived uneventfully. All titanium rings were exposed three weeks after surgery. Two implants were lost due to infection caused by exposure of the titanium ring in the holding group, but no implants were lost or loosened, and no signs of infection were observed in the removal group. The remaining six implants in the holding group were stable with titanium ring exposure, and the eight implants in the removal group were covered by gingival tissue.

\section{Bone augmentation height (BAH) measurement}

The distance between the implant shoulder and the bottom of the surrounding bone was assessed at the buccal and lingual regions by using Vernier calipers (accuracy $0.02 \mathrm{~mm}$ ) (Fig. 1). Negative values were given if the top of the buccal and lingual bone was above the implant shoulder. This, in turn, meant that more bone quantity was acquired for higher negative values. In each implant, three repetitions were performed to calculate the mean value for reducing the deviation, and buccal and lingual BAH with the augmented areas of the circumferential defects was defined as $3 \mathrm{~mm}$ minus the calculated mean value. 
Median values of BAH were measured (in $\mathrm{mm}$ ) to be $2.46(2.18-2.62) \mathrm{mm}$ in buccal of the $\mathrm{R}$ group, 2.8 (2.66-3.02) $\mathrm{mm}$ in lingual of the removal group, $0.64(0.32-0.92) \mathrm{mm}$ in buccal of the holding group, and $0.7(0.22-1.22) \mathrm{mm}$ in lingual of the holding group. The difference between $\mathrm{BAH}$ was statistically significant in the buccalingual aspects of the implants in the two groups $(P<0.001)$. The removal group exhibited a higher $\mathrm{BAH}$ than the holding group $(P<0.001)$. Lingual significantly increased $\mathrm{BAH}$ in contrast to buccal BAH in the R group $(P=0.002)$. Nevertheless, comparing the buccal and lingual regions in the holding group, the difference in BAH was not statistically significant $(P=0.374)$ (Table 1) (Fig. 2).

Table 1

Buccal and Lingual Bone Augmentation Height (BAH) of Titanium Ring Technology with Primary Wound Dehiscence (mm)

\begin{tabular}{|llllll|}
\hline Group & Side & Median & Minimum & Maximum & P values \\
\hline R group & Buccal & 2.46 & 2.18 & 2.62 & Total: $P<0.001$ \\
\cline { 2 - 5 } & Lingual & 2.80 & 2.66 & 3.02 & R vs. H: $P<0.001$ \\
H group & Buccal & 0.64 & 0.32 & 0.92 & BR vs. LR: $P=0.002$ \\
& Lingual & 0.70 & 0.22 & 1.22 & BH vs. LH: $P=0.374$
\end{tabular}

R group: the titanium rings removed group. $\mathrm{H}$ group: the titanium rings holding group. $\mathrm{BR}=\mathrm{Buccal}$ of $\mathrm{R}$ group; $\mathrm{LR}=$ Lingual of $\mathrm{R}$ group; $\mathrm{BH}=$ Buccal of $\mathrm{H}$ group; $\mathrm{LH}=$ Lingual of $\mathrm{H}$ group.

\section{Micro-CT analysis}

Due to severe infection and poor bone augmentation in the holding group, only 8 bone sample blocks of the removal group were analyzed by micro-CT and histology.

The micro-CT image displayed obvious bone resorption in the buccal bone augmentation area, which led to a buccal bone width of less than $2 \mathrm{~mm}$ (Fig. 3). A high level of trabecular bone details was found in the reconstructed 3D CT image, which showed the distinct structures of the native and newly formed bone around the titanium implants. In the native bone region, the cancellous bone structure showed large, rodlike, connected trabeculae with fusiform or oval voids in between. However, there were also some areas of incompletely matured bone with a disorganized structure comprised of thin and incompact trabeculae, which were mostly observed near the implant shoulder (Fig. 4).

As seen in Table 2, the BV value of V1 was between 69.67 and 84.39 in the R group, with a median of 76.81. In addition, the BV/TV values were all more than $70 \%$ for all implants in the $\mathrm{R}$ group, showing a predictable vertical bone augmentation effect. In the analysis of the BS/TV, the median and range were 3.16 and 3.06-3.83, respectively, which indirectly reflects the bone augmentation effect of the titanium ring. Furthermore, the BMD results showed that there was a significant difference between V1 and V2 ( $P$ $=0.004)$. 
Table 2

Values of BV, BV/TV, BS/TV and BMD for V1 and BMD for V2, as measured by micro-CT in the R group

\begin{tabular}{|lllll|}
\hline Region & Outcome & Median & Maximum & Minimum \\
\hline V1 & BV $\left(\mathrm{mm}^{\wedge} 3\right)$ & 76.81 & 84.39 & 69.67 \\
\cline { 2 - 5 } & BV/TV $(\%)$ & 80.07 & 87.98 & 72.64 \\
\cline { 2 - 5 } & BS/TV $\left(\mathrm{mm}^{\wedge}-1\right)$ & 3.16 & 3.83 & 3.06 \\
\cline { 2 - 5 } V2 & BMD $\left(\mathrm{g} / \mathrm{cm}^{\wedge} 3\right)$ & 1.46 & 1.57 & 1.39 \\
\hline
\end{tabular}

V1: The bone augmentation region corresponding to the dimensions of the titanium ring. V2: The native bone under V1 with a diameter of $7.2 \mathrm{~mm}$ and a height of $5 \mathrm{~mm}$.

\section{Histological observation}

In general, all samples in the removal group showed ideal osseointegration over the surface of the implant and no signs of inflammation in the area of the implant. The structure of the graft material was either completely replaced by newly formed cancellous bone or in the process of intensive bone remodeling. Good bone healing and consolidation were observed in very dense bone. The new bone, which was near the superior border of the implant in the lingual region, showed signs of minor resorption in the removal group (Fig. 5).

\section{Discussion}

In the present study, 16 mandible alveolar surgical sites were vertically augmented using a titanium ring with a 1:1 mixture of autogenous bone particles and DBBM in beagle dogs. The titanium ring was derived from titanium mesh. The application of titanium mesh in terms of GBR techniques has received increasing attention due to reports that document predictable and consistent results with this material for 3-dimensional bone reconstruction, whether using delayed or simultaneous implantation ${ }^{31}$. Titanium mesh has good mechanical and biological properties. Its high strength and stiffness maintain a highly stable space support for regenerating bone and tissue integration ${ }^{22}$, and its low electrical conductivity and noncytotoxicity show high and persistent corrosion resistance and no effect on cell proliferation 32,17 . Apparently, as a kind of barrier membrane, titanium mesh can satisfy the basic principle of GBR ${ }^{14}$.

The key factor for the success of GBR with titanium mesh is the stabilization of bone grafts beneath the membrane ${ }^{25}$. Specifically, as blood clot formation is the start of the bone healing process and the successful osseointegration of titanium implants ${ }^{33}$, the interaction between the blood clot and the rough titanium surface induces early cell recruitment ${ }^{34}$. A stable blood clot between the broken bone ends is considered one of the most important factors for guaranteeing the integrity of the blood vessel and supporting subsequent granulation tissue formation after implantation ${ }^{35}$. The fixation methods of 
titanium mesh mainly include suture fixation and titanium screw fixation ${ }^{17}$. However, the retention of sutures is relatively weak, and movement of the titanium mesh may occur due to surrounding stress [36]. In addition, the effect of titanium screw placement on implantation and additional incisions for removing the titanium mesh and screws in subsequent surgery will increase the patient's trauma and the difficulty of the surgery ${ }^{30,37}$. In the present study, a new type of retaining screw was used to fix the titanium ring on the implant, which exhibited acceptable osteogenic responses.

Vertical bone augmentation with simultaneous implant placement using a titanium ring results in a substantial shortening of the overall treatment but may produce complications in the form of postoperative wound dehiscence as in the present study. Similar results can be observed with titanium mesh. It is thought that when vertical ridge augmentation is performed, the risk of exposure of the titanium mesh is high ${ }^{38-40}$. Early exposure of the bone graft to the oral cavity and exposure occurring within 4 weeks after bone augmentation have been identified as reasons for infection and bone graft loss, and the soft tissue associated with inflammation remains in place and impedes the ingrowth of bone even after the resolution of the inflammation ${ }^{17,41}$. However, other studies have reported that premature exposure to titanium mesh did not result in either infection or decreased bone augmentation ${ }^{19,42}$. In the present study, all of the titanium rings were exposed to the oral cavity at 3 weeks, and no infection or purulence was detected, although wound dehiscence was detected within the first month. Previous authors have reported similar results, that premature exposure did not result in infection of the treated sites or decreased bone formation ${ }^{42,43}$. Nevertheless, several other investigators have demonstrated that "premature" exposure had a more significant influence on new bone formation than late exposure ${ }^{18,44,45}$. In any case, tension-free sutures without any blanching in the suture line or over the graft, improvement of the barrier membrane and adequate periosteal scoring are the most effective solutions to prevent titanium mesh exposure $30,42,46$. In this study, the titanium ring produced by $3 \mathrm{D}$ printing had sharp edges, which might be the cause of the postoperative exposure ${ }^{47}$. In addition, the rough surface of the titanium ring was susceptible to bacterial contamination and could have caused mechanical irritation of the mucosal flaps ${ }^{48,49}$.

Currently, the management of titanium mesh exposure varies. A clinical retrospective study reported that the titanium mesh should be removed as soon as possible, and subsequent anti-infection operations should be conducted if early exposure occurs ${ }^{50}$. In contrast, the mean values reported in a systematic review found that when titanium mesh exposure occurred, mesh removal was necessary in only $20 \%$ of the cases ${ }^{51}$. Hartmann et al. ${ }^{52}$, in a clinical study, first classified the postexposure titanium mesh according to the area of mesh exposed and verified the effect of each treatment. The authors suggested premature removal of the mesh, and consequent soft-tissue healing was necessary when a large area of mesh exposure appeared. In the present study, the postexposure titanium rings were removed 4 weeks after the operation in the removal group.

The process of osseointegration can be summarized as follows: formation of a coagulum and granulation tissue and then bone formation and remodeling ${ }^{53}$. In in vivo studies, hard tissue is present 
around the Bio-Oss at 4 weeks because the bone grafts can be gradually replaced by newly formed bone ${ }^{54,55}$. The new bone includes woven bone often combined with both parallel-fibered and lamellar bone at 4 weeks ${ }^{56}$. After 12 weeks of micro-CT analysis in this study, the percentage of new bone formation was observed to range from $72.64-87.98 \%$ in the removal group. Furthermore, pseudoperiosteum and ingrowth of soft tissue were not detected at the treated sites, which may be related to the removal of the titanium ring and needs more experimental evidence for further confirmation. The samples in this study were collected at 3 months postoperatively. However, the BMD of V1 was lower than that of V2, which showed that calcium deposition in osteoids requires additional time.

From the analysis of the BAH, interesting observations were made. The lingual alveolar bone height was acquired at an ideal level ${ }^{57}$. However, obvious bone resorption was found on the buccal form micro-CT. In our experience, titanium ring deficiency may cause bone grafts and immature bone to be affected by perioral buccal muscle pressure during the healing period, which causes stress bone resorption instead of inflammatory bone resorption in the removal group ${ }^{58}$. In the holding group, the treatment site without removal of the titanium ring was exposed to the oral cavity for a long time and caused severe inflammation. The implant was loose or lost as a result of osseointegration failure and peri-implant bone loss. Thus, similar to titanium mesh, titanium ring exposure did not directly result in significant bone resorption ${ }^{59}$. All pores of the titanium ring were designed on the crest in this study, which allowed bacteria to easily enter once it was exposed.

The data have to be explained with caution because the design of this study allowed only an explorative statistical analysis and a basis for future studies. Some limitations need to be taken into account regarding this animal study. Future studies should improve the design of the titanium rings and their surface preparation to prevent titanium exposure in order to evaluate the success of titanium rings.

\section{Conclusions}

Within the study limitations, the use of titanium rings retained in implants was found to be a reliable alternative method for vertical bone augmentation, and removal of the titanium rings is better for bone augmentation when early exposure of titanium rings occurs.

\section{Abbreviations}

BAH: Bone augmentation height

GBR: Guided bone regeneration

e-PTFE: Expanded-polytetrafluoroethylene

3D: Three-dimensional

DBBM: Deproteinized natural bovine bone mineral 
micro-CT: Computed microtomography

ROI: Region of interest

BV: Bone volume

BV/TV: Bone volume/total volume

BS/TV: Bone surface/total volume

BMD: Bone mineral density

\section{Declarations}

\section{Acknowledgments}

Not applicable

\section{Authors' contributions}

The author K.Y. conceived the ideas and designed this study. K.Y., Q.Q.L., J.Y., L.J.L., P.Z.P. and M.Y.Z. did the animal experiment. Q.Q.L. and J.Y. performed the micro-CT and histological staining. P.Z.P. and M.Y.Z. performed the measurements. Q.Q.L., J.Y. and L.J.L. statistically analyzed the data. K.Y., Q.Q.L. and J.Y. critically revised and guided the scientific writing. All authors meet the ICMJE criteria for authorship.

Q.Q.L. and J.Y. contributed equally to this work.

\section{Competing interests}

The authors declare no competing interests.

\section{Data availability}

The datasets generated during and/or analysed during the current study are available from the corresponding author on reasonable request.

\section{Ethics declarations}

This animal experiment was approved by the Animal Ethics Committee of Southwest Medical University (reference number SWMU20210372).

\section{Consent to publish}

Manuscript is approved by all authors for publication

\section{Funding}


The study was funded by Innovation and Entrepreneurship Training program for College students (Ref. S201910653022)

\section{Availability of data and materials}

The datasets used and/or analyzed during the current study are available from the corresponding author on reasonable request.

\section{Supplementary Information}

Not applicable

\section{References}

1. Lekovic, V. et al. Preservation of alveolar bone in extraction sockets using bioabsorbable membranes.J Periodontol. 69(9), 1044-1049(1998).

2. Tolstunov, L., Hamrick, J., Broumand, V., Shilo, D. \& Rachmiel, A. Bone Augmentation Techniques for Horizontal and Vertical Alveolar Ridge Deficiency in Oral Implantology.ral Maxillofac Surg Clin North Am. 31(2), 163-191(2019).

3. McAllister, B. S. \& Haghighat, K. Bone augmentation techniques.J Periodontol.78(3),377-396(2007).

4. Aghaloo, T. L. \& Moy, P. K. Which hard tissue augmentation techniques are the most successful in furnishing bony support for implant placement?.Int J Oral Maxillofac Implants.22 Suppl,49-70( 2007).

5. Esposito, M. et al. The efficacy of horizontal and vertical bone augmentation procedures for dental implants - a Cochrane systematic review. Eur J Oral Implantol. 2(3),167-184(2009).

6. Triplett, R. G. \& Schow, S. R. (1996). Autologous bone grafts and endosseous implants: complementary techniques. J Oral Maxillofac Surg. 54(4), 486-494 (1996).

7. Keestra, J. A., Barry, O., Jong, L. \& Wahl, G. Long-term effects of vertical bone augmentation: a systematic review.J Appl Oral Sci. 24(1),3-17(2016).

8. Hameed, M. H., Gul, M., Ghafoor, R. \& Khan, F. R. Vertical Ridge Gain with Various Bone Augmentation Techniques: A Systematic Review and Meta-Analysis.J Prosthodont. 28(4),421-427(2019).

9. Giesenhagen, B., Martin, N., Jung, O. \& Barbeck, M. Bone Augmentation and Simultaneous Implant Placement with Allogenic Bone Rings and Analysis of Its Purification Success. Materials (Basel). 12(8), 1291(2019).

10. Polo, W. C., Cury, P. R., Sendyk, W. R. \& Gromatzky, A. Posterior mandibular alveolar distraction osteogenesis utilizing an extraosseous distractor: a prospective study.J Periodontol. 76(9),14631468(2005).

11. Robiony, M., Polini, F., Costa, F. \& Politi, M. Osteogenesis distraction and platelet-rich plasma for bone restoration of the severely atrophic mandible: preliminary results.J Oral Maxillofac Surg. 60(6),630635(2002). 
12. McAllister, B. S. Histologic and radiographic evidence of vertical ridge augmentation utilizing distraction osteogenesis: 10 consecutively placed distractors. J Periodontol, 72 (12), 1767-1779 (2001).).

13. Sakkas, A., Wilde, F., Heufelder, M., Winter, K. \& Schramm, A. Autogenous bone grafts in oral implantology-is it still a "gold standard"? A consecutive review of 279 patients with 456 clinical procedures. Int J Implant Dent. 3(7),23(2017).

14. Urban, I. A., Montero, E. \& Monje, A. \& Sanz-Sánchez, I. Effectiveness of vertical ridge augmentation interventions: A systematic review and meta-analysis. J Clin Periodontol. 46 Suppl 21,319-339( 2019).

15. Boyne, P. J., Cole, M. D., Stringer, D. \& Shafqat, J. P. A technique for osseous restoration of deficient edentulous maxillary ridges.J Oral Maxillofac Surg. 43(2),87-91(1985).

16. Degidi, M., Scarano, A. \& Piattelli, A. Regeneration of the alveolar crest using titanium micromesh with autologous bone and a resorbable membrane.J Oral Implantol.29(2),86-90(2003).

17. Xie, Y., Li, S., Zhang, T., Wang, C. \& Cai, X. Titanium mesh for bone augmentation in oral implantology: current application and progress.Int J Oral Sci.12(1),37(2020).

18. Cucchi, A., Vignudelli, E., Napolitano, A., Marchetti, C. \& Corinaldesi, G. Evaluation of complication rates and vertical bone gain after guided bone regeneration with non-resorbable membranes versus titanium meshes and resorbable membranes. A randomized clinical trial.Clin Implant Dent Relat Res.19(5),821-832(2017).

19. von Arx, T., Hardt, N. \& Wallkamm, B. The TIME technique: a new method for localized alveolar ridge augmentation prior to placement of dental implants. Int J Oral Maxillofac Implants, 11(3),387-394( 1996).

20. Roccuzzo, M., Ramieri, G., Bunino, M. \& Berrone, S. Autogenous bone graft alone or associated with titanium mesh for vertical alveolar ridge augmentation: a controlled clinical trial.Clin Oral Implants Res. 18(3),286-294(2007).

21. Miller, R. J., Korn, R. J. \& Miller, R. J. Indications for Simultaneous Implantation and Bone Augmentation Using the Allograft Bone Ring Technique.Int J Periodontics Restorative Dent. 40(3),345-352(2020).

22. Rakhmatia, Y. D., Ayukawa, Y., Furuhashi, A. \& Koyano, K. Microcomputed tomographic and histomorphometric analyses of novel titanium mesh membranes for guided bone regeneration: a study in rat calvarial defects. Int J Oral Maxillofac Implants.29(4),826-835(2014).

23. Her, S., Kang, T. \& Fien, M. J. Titanium mesh as an alternative to a membrane for ridge augmentation.J Oral Maxillofac Surg.70(4),803-810(2012).

24. Torres, J. et al. Platelet-rich plasma may prevent titanium-mesh exposure in alveolar ridge augmentation with anorganic bovine bone.J Clin Periodontol.37(10),943-951(2010).

25. Rakhmatia, Y. D., Ayukawa, Y., Furuhashi, A. \& Koyano, K. Current barrier membranes: titanium mesh and other membranes for guided bone regeneration in dental applications.J Prosthodont Res. 57(1),3-14(2013). 
26. Ciocca, L. et al. Prosthetically CAD-CAM-Guided Bone Augmentation of Atrophic Jaws Using Customized Titanium Mesh: Preliminary Results of an Open Prospective Study.J Oral Implantol.44(2), 131-137(2018).

27. Hartmann, A. \& Seiler, M. Minimizing risk of customized titanium mesh exposures - a retrospective analysis.BMC Oral Health. 20(7),36(2020).

28. Buser, D., Dula, K., Hirt, H. P. \& Schenk, R. K. Lateral ridge augmentation using autografts and barrier membranes: a clinical study with 40 partially edentulous patients.J Oral Maxillofac Surg. 54(4),420433(1996).

29. Simion, M., Jovanovic, S. A., Trisi, P., Scarano, A. \& Piattelli, A. Vertical ridge augmentation around dental implants using a membrane technique and autogenous bone or allografts in humans. Int $J$ Periodontics Restorative Dent. 18(1),8-23(1998).

30. Atef, M., Tarek, A., Shaheen, M., Alarawi, R. M. \& Askar, N. Horizontal ridge augmentation using native collagen membrane vs titanium mesh in atrophic maxillary ridges: Randomized clinical trial. Clin Implant Dent Relat Res.22(2), 156-166(2020).

31. Briguglio, F. et al. The Use of Titanium Mesh in Guided Bone Regeneration: A Systematic Review. Int J Dent. 2019, 9065423 (2019).

32. .Cordeiro, J. M. \& Barão, V. Is there scientific evidence favoring the substitution of commercially pure titanium with titanium alloys for the manufacture of dental implants?.Mater Sci Eng C Mater Biol Appl.71,1201-1215(2017).

33. Shiu, H. T., Goss, B., Lutton, C., Crawford, R. \& Xiao, Y. Formation of blood clot on biomaterial implants influences bone healing. Tissue Eng Part B Rev.20(6),697-712(2014).

34. Yang, J., Zhou, Y., Wei, F. \& Xiao, Y. Blood clot formed on rough titanium surface induces early cell recruitment.Clin Oral Implants Res.27(8), 1031-1038(2016).

35. Kolar, P. et al. The early fracture hematoma and its potential role in fracture healing. Tissue Eng Part $B$ Rev.16(4),427-434(2010).

36. Li, S. et al. A novel digital and visualized guided bone regeneration procedure and digital precise bone augmentation: A case series.Clin Implant Dent Relat Res.23(1),19-30.(2021).

37. Zhang, T., Zhang, T. \& Cai, X. The application of a newly designed L-shaped titanium mesh for GBR with simultaneous implant placement in the esthetic zone: $A$ retrospective case series study.Clin Implant Dent Relat Res. 21(5),862-872(2019).

38. von Arx, T. \& Kurt, B. Implant placement and simultaneous ridge augmentation using autogenous bone and a micro titanium mesh: a prospective clinical study with 20 implants. Clin Oral Implants Res. 10(1),24-33(1999).

39. Maiorana, C. et al. Clinical and Radiographic Evaluation of Simultaneous Alveolar Ridge Augmentation by Means of Preformed Titanium Meshes at Dehiscence-Type Peri-Implant Defects: A Prospective Pilot Study.Materials (Basel). 13(10),2389(2020).

40. Soldatos, N. K. et al. Limitations and options using resorbable versus nonresorbable membranes for successful guided bone regeneration. Quintessence Int. 48(2),131-147(2017). 
41. Yeo, A. et al. Lateral ridge augmentation using a PCL-TCP scaffold in a clinically relevant but challenging micropig model.Clin Oral Implants Res.23(12),1322-1332(2012).

42. Pinho, M. N. et al. Titanium membranes in prevention of alveolar collapse after tooth extraction.Implant Dent. 15(1),53-61(2006).

43. Paquay, Y. C., de Ruijter, J. E., van der Waerden, J. P. \& Jansen, J. A. Wound healing phenomena in titanium fibre mesh: the influence of the length of implantation.Biomaterials.18(2),161-166(1997).

44. Machtei, E. E. The effect of membrane exposure on the outcome of regenerative procedures in humans: a meta-analysis. J Periodontol, 72 (4), 512-516 (2001).).

45. Moses, O., Pitaru, S., Artzi, Z. \& Nemcovsky, C. E. Healing of dehiscence-type defects in implants placed together with different barrier membranes: a comparative clinical study. Clin Oral Implants Res.16(2),210-219(2005).

46. Watzinger, F. et al. Guided bone regeneration with titanium membranes: a clinical study.Br J Oral Maxillofac Surg. 38(4),312-315(2000).

47. Becker, W. et al. A prospective multi-center study evaluating periodontal regeneration for Class II furcation invasions and intrabony defects after treatment with a bioabsorbable barrier membrane: 1year results.J Periodontol. 67(7),641-649(1996).

48. Louis, P. J., Gutta, R., Said-Al-Naief, N. \& Bartolucci, A. A. Reconstruction of the maxilla and mandible with particulate bone graft and titanium mesh for implant placement.J Oral Maxillofac Surg. 66(2),235-245(2008).

49. Chouirfa, H., Bouloussa, H., Migonney, V. \& Falentin-Daudré, C. Review of titanium surface modification techniques and coatings for antibacterial applications.Acta Biomater.83,37-54(2019).

50. Corinaldesi, G., Pieri, F., Sapigni, L. \& Marchetti, C. Evaluation of survival and success rates of dental implants placed at the time of or after alveolar ridge augmentation with an autogenous mandibular bone graft and titanium mesh: a 3- to 8-year retrospective study.Int J Oral Maxillofac Implants. 24(6), 1119-1128(2009).

51. Rasia-dal Polo, M., Poli, P. P., Rancitelli, D., Beretta, M. \& Maiorana, C. Alveolar ridge reconstruction with titanium meshes: a systematic review of the literature.Med Oral Patol Oral Cir Bucal. 19(6),e639-e646(2014).

52. Hartmann, A., Hildebrandt, H., Schmohl, J. U. \& Kämmerer, P. W. Evaluation of Risk Parameters in Bone Regeneration Using a Customized Titanium Mesh: Results of a Clinical Study.Implant Dent. 28(6),543-550(2019).

53. Bosshardt, D. D., Chappuis, V. \& Buser, D. Osseointegration of titanium, titanium alloy and zirconia dental implants: current knowledge and open questions. Periodontol 2000. 73(1), 22-40 (2017).

54. Kasuya, S. New Bone Formation Process Using Bio-Oss and Collagen Membrane for Rat Calvarial Bone Defect: Histological Observation. Implant Dent. 27(2),158-164(2018).

55. Berglundh, T., Abrahamsson, I., Lang, N. P. \& Lindhe, J. De novo alveolar bone formation adjacent to endosseous implants. Clin Oral Implants Res.14(3),251-262(2003). 
56. Esfahanizadeh, N., Daneshparvar, P., Takzaree, N., Rezvan, M. \& Daneshparvar, N. Histologic Evaluation of the Bone Regeneration Capacities of Bio-Oss and MinerOss X in Rabbit Calvarial Defects. Int J Periodontics Restorative Dent.39(6),e219-e227(2019).

57. Yu, K. et al. Evaluation of Resorption and Osseointegration of Autogenous Bone Ring Grafting in Vertical Bone Defect With Simultaneous Implant Placement in Dogs.J Oral Implantol.47(4),295-302( 2021).

58. Klijn, R. J., Meijer, G. J., Bronkhorst, E. M. \& Jansen, J. A. A meta-analysis of histomorphometric results and graft healing time of various biomaterials compared to autologous bone used as sinus floor augmentation material in humans. Tissue Eng Part B Rev. 16(5),493-507(2010).

59. von Arx, T., Wallkamm, B. \& Hardt, N. Localized ridge augmentation using a micro titanium mesh: a report on 27 implants followed from 1 to 3 years after functional loading. Clin Oral Implants Res.9(2), 123-130(1998).

\section{Figures}

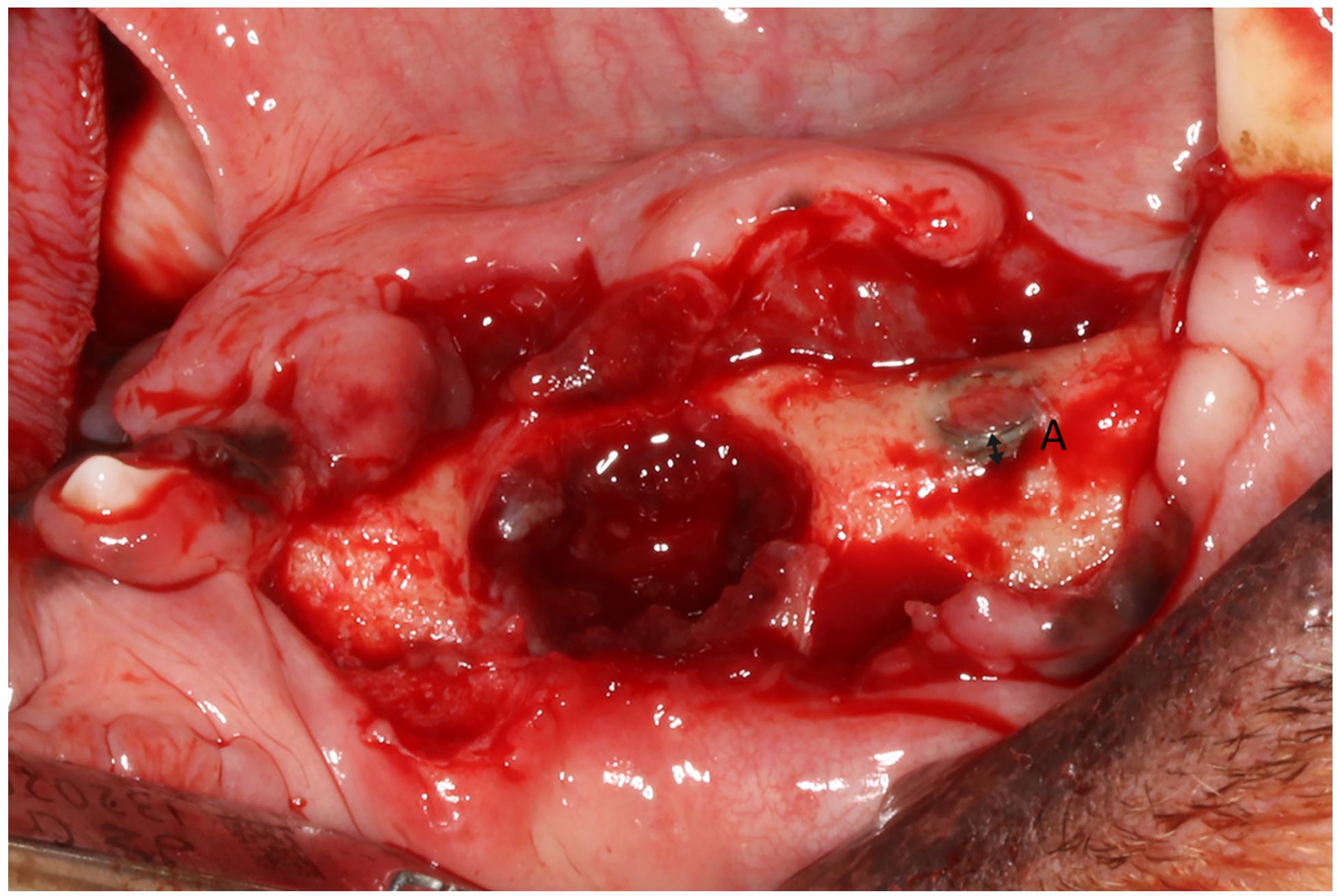

\section{Figure 1}

a The distance between the implant shoulder and the bottom of the surrounding bone. 


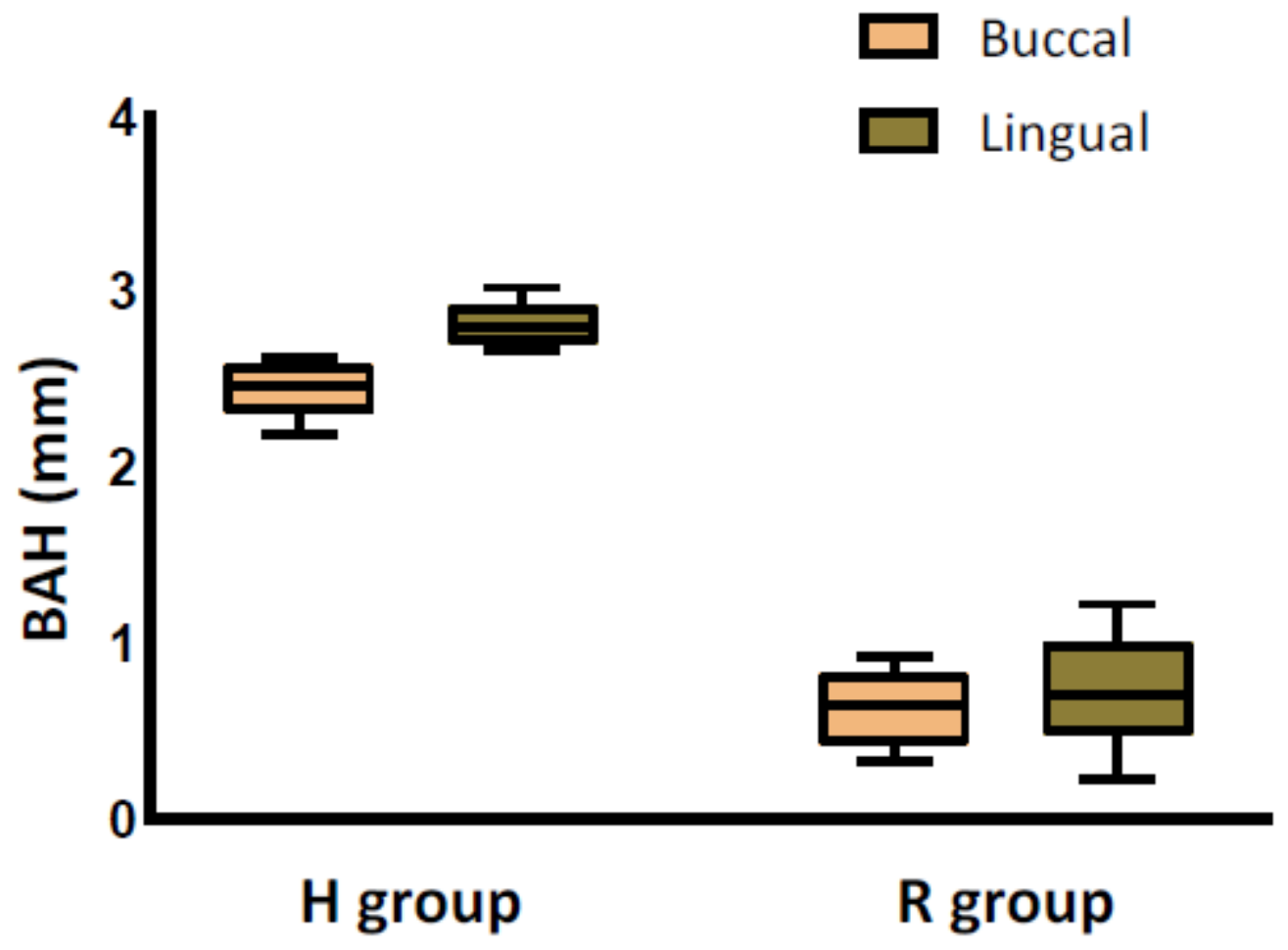

Figure 2

Box plots for the distribution of the buccal and lingual bone augmentation height. Horizontal bars designate median values. Boxes delineate median plus 1 st and 3rd quartile (IQR), and error bars show the upper and lower adjacent values (the most extreme values within $1.5 \times$ IQR). 


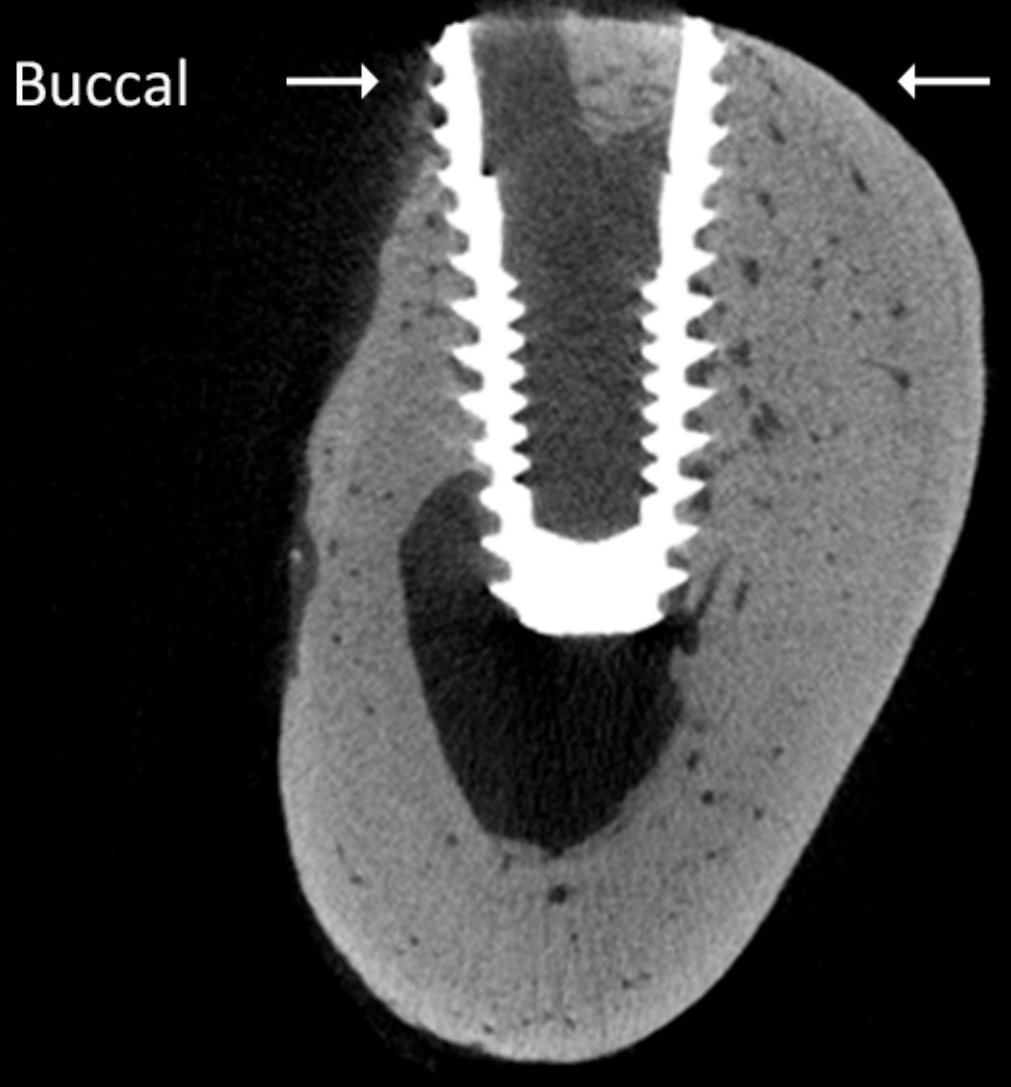

Lingual

CT value

$-385.00$

2016.33

4597.67

7089.00

$2 \mathrm{~mm}$

Figure 3

Micro-CT image of the implant with surrounding bone in the coronal plane. 


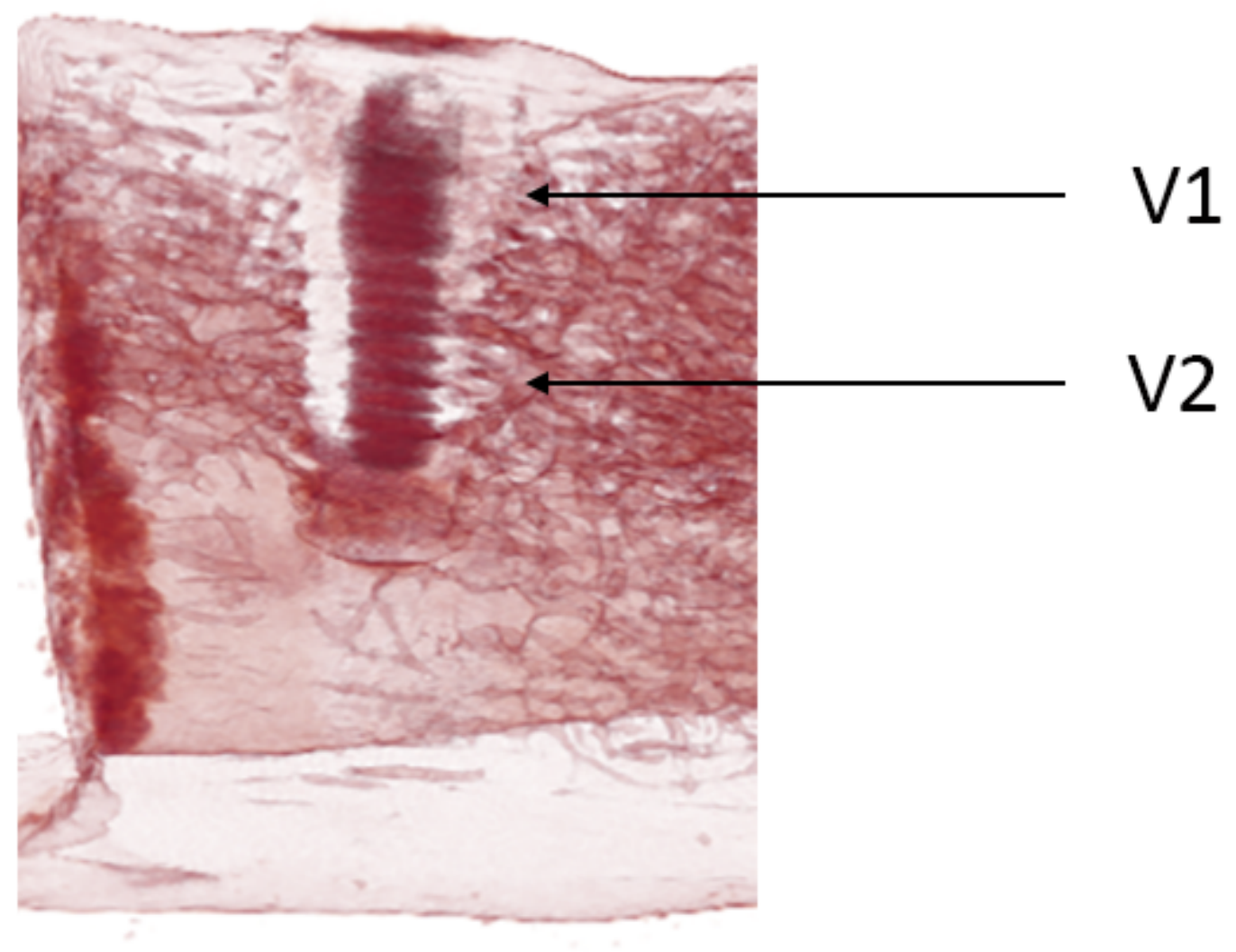

$2 \mathrm{~mm}$

Figure 4

$3 \mathrm{D}$ reconstruction micro-CT image in the sagittal plane. 

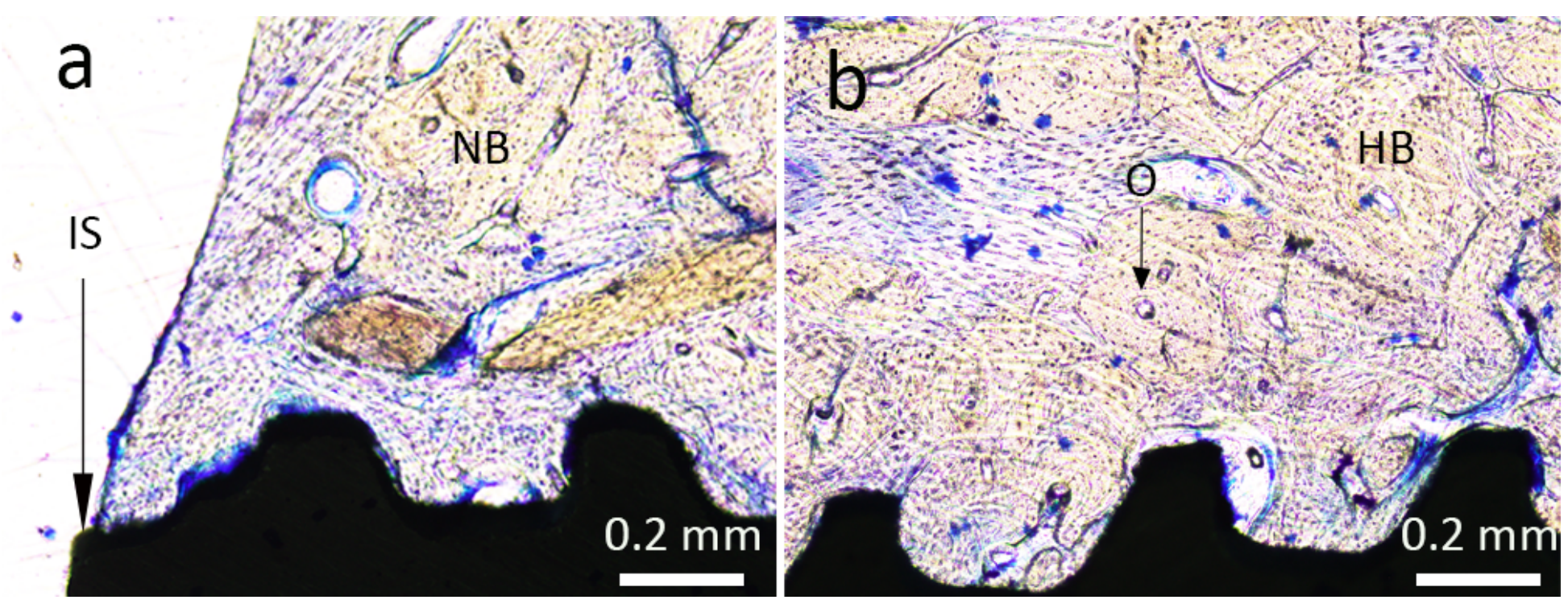

\section{Figure 5}

Representative buccolingual histologic sections of vertical bone defects after the placement of bone lever tapered implants and simultaneous augmentation with a titanium ring. IS = implant shoulder; NB = new bone (a); HB = host bone; $\mathrm{O}$ = osteon (b). Stained with toluidine blue.
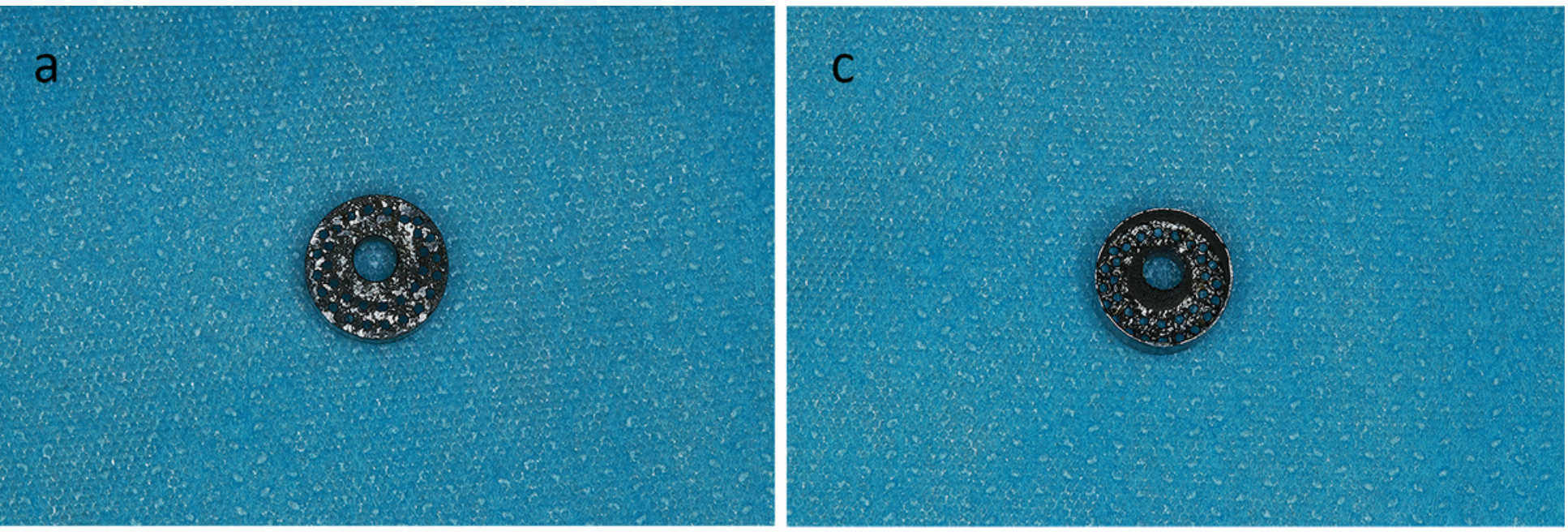

b 
The design of the titanium ring and retaining screw in the present study. (a) The crest of the titanium ring. (b) The profile of the titanium ring. (C) The bottom surface of the titanium ring. (d) Retaining screw.

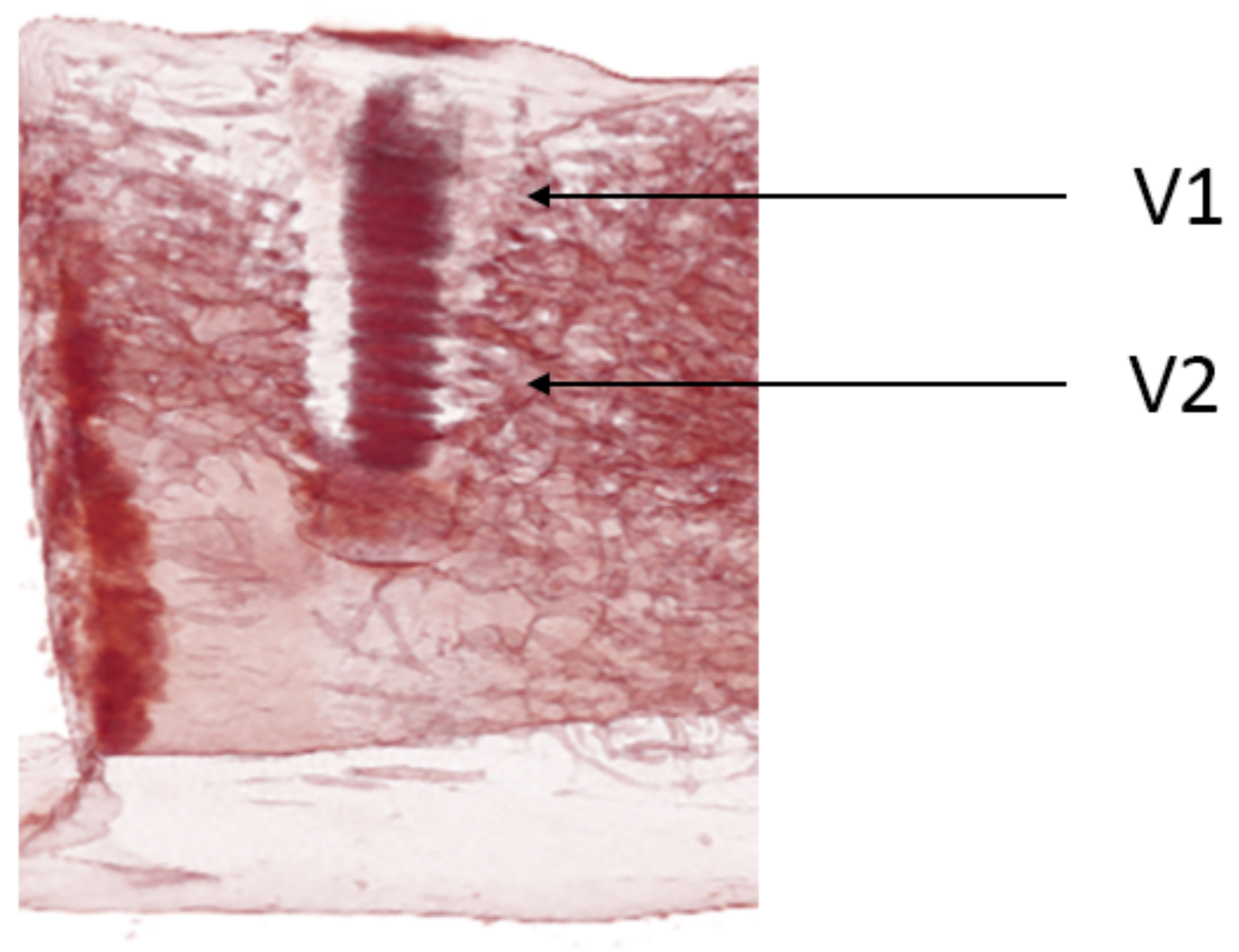

\section{$2 \mathrm{~mm}$}

\section{Figure 7}

The design of the titanium ring in the present study, which is fixed on the implant by the retaining screw. The height, diameter and thickness of the titanium ring were $3.5 \mathrm{~mm}, 7.2 \mathrm{~mm}$ and $0.1 \mathrm{~mm}$, respectively. In addition, the osteogenesis space between the titanium ring and the implant was $0.4 \mathrm{~mm}$ above, $2 \mathrm{~mm}$ in the buccal space and $1.4 \mathrm{~mm}$ in the lingual space. 

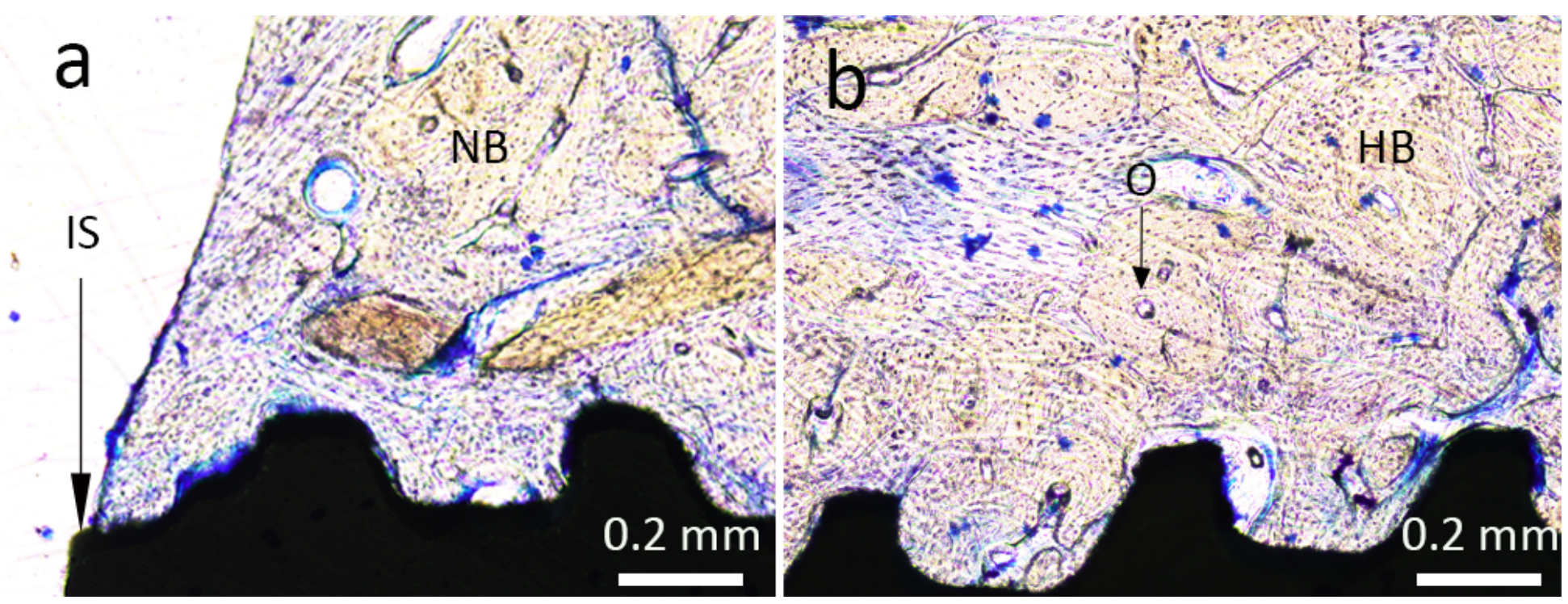

Figure 8

Intraoperative view of the treated area. (a) Two vertical ridge defects $(7.2 \times 7.2 \times 3.5 \mathrm{~mm})$ were created on each side of the mandible. (b) Placement of implants. (c) Only the titanium ring is installed on the left, and the retaining screw fixes the titanium ring on the implant on the right. (d) The titanium ring was exposed 3 weeks after surgery. 